\title{
On the Analysis of Discrete Epidemic Models with Specific Nonlinear Incidence Rate
}

\author{
Amine BOUAINE ${ }^{1}$ \\ Faculty of sciences Ben M'Sik \\ Department Maths \& Computer Sciences \\ Laboratory Analysis Modeling and simulation \\ Hassan II Mohammedia University \\ Casablanca, Morocco \\ lamine.bouaine@gmail.com
}

\author{
Mostafa RACHIK ${ }^{2}$, Khalid HATTAF ${ }^{3}$ \\ Faculty of sciences Ben M'Sik \\ Department Maths \& Computer Sciences \\ Laboratory Analysis Modeling and simulation \\ Hassan II Mohammedia University \\ Casablanca, Morocco \\ ${ }^{2}$ m_rachik@yahoo.fr, ${ }^{3}$ k.hattaf@yahoo.fr
}

\begin{abstract}
The aim of this paper is to study the local stability of discrete epidemic models with specific nonlinear incidence rate. So, we establish criteria on local stability of the disease-free equilibrium, furthermore, using Schur-Cohn Criterion, important criterion on local stability of endemic equilibrium is also determined.
\end{abstract}

Keywords: Biomathematics, Stability, Discrete SIR epidemic model, Reproduction number, Epidemiology.

\section{INTRODUCTION}

The discrete time models are more convenient to describe epidemics than the continuous ones. On the one hand, the statistic data concerning diseases is collected in discrete time, on the other hand, the discrete-time models may escape some mathematical complexities like regularity of solutions, adequate topology and the choice of state space. Furthermore, the numerical simulations of continuous time models are obtained by the way of discretization.

In literature, a huge variety of continuous-time models have been developed in order to understand disease transmission dynamics and to study epidemiological processes [1-3]. Y.Enatsu [4] apply a variation of Euler backward discretization in order to study a discrete SIR epidemic model with a class of nonlinear incidence rate and a distributed latent period. S.Jang [5] proposes and analyses a simple discrete-time West Nile epidemic model. The derivation of this model is based on a continuous-time model proposed by Cruz-Pacheco et al [6]. S.Jang and S.Elaydi [7] obtain systems of difference equations from the continuous time model by using nonstandard discretization technique. They make comparisons between the continuous-time model and its discrete counterpart.

This paper puts the stress on different important issues and provides many advantages. Firstly, we study a discrete SIR epidemic model (1) with specific nonlinear incidence rate $\beta S_{k} I_{k} /\left(1+\alpha_{1} S_{k}+\alpha_{2} I_{k}+\alpha_{3} S_{k} I_{k}\right)$, where $\beta, \alpha_{1}, \alpha_{2}$ and $\alpha_{3} \geq 0$ are constants. $S_{k}$ is the number of susceptible at time $\mathrm{k}$, whereas $\mathrm{I}_{\mathrm{k}}$ is the number of infected at time $\mathrm{k}$. It is very important to note that this incidence rate becomes the bilinear incidence rate if $\alpha_{1}=\alpha_{2}=\alpha_{3}=0$, the saturated incidence rate if $\alpha_{1}=\alpha_{3}=0$ or $\alpha_{2}=\alpha_{3}=0$, the modified saturated incidence rate proposed in $[8,9]$ when $\alpha_{3}=0$, and Crowley-Martin functional response presented in [10-12] if $\alpha_{3}=\alpha_{1} \alpha_{2}$. Secondly, this model is studied in discrete-time, is more accurate and more convenient as we explained above. Thirdly, we set rigid criteria on local stability in two cases: disease free equilibrium and endemic equilibrium.

This paper is organized as follows. In the next section, we present a discrete SIR epidemic model with specific nonlinear incidence rate, its basic reproduction number and its jacobian matrix in order to study stability of the epidemic model. The section 3 puts the stress on stability of disease free equilibrium. In the section 4, we rely on Schur-Cohn Criterion to study stability of endemic equilibrium. Finally, conclusions are summarized in section 5 . 


\section{STABILITY OF THE EPIDEMIC MODEL}

We consider a discrete SIR epidemic model. The population is divided into three disease-state compartments: susceptible individuals (S), people who can catch the disease; infectious (infective) individuals (I), people who have the disease and can transmit the disease; recovered individuals $(\mathrm{R})$, people who have recovered from the disease. We assume that an individual can be infected only through contacts with infectious individuals and that immunity is permanent. Our model is described as follows

$$
\left\{\begin{array}{l}
S_{k+1}=\Lambda-(\mu-1) S_{k}-\frac{\beta S_{k} I_{k}}{1+\alpha_{1} S_{k}+\alpha_{2} I_{k}+\alpha_{3} S_{k} I_{k}} \\
I_{k+1}=\frac{\beta S_{k} I_{k}}{1+\alpha_{1} S_{k}+\alpha_{2} I_{k}+\alpha_{3} S_{k} I_{k}}-(\mu+d+r-1) I_{k} \\
R_{k+1}=-(\mu-1) R_{k}+r I_{k}
\end{array}\right.
$$

With $S_{0} \geq 0, I_{0} \geq 0$ and $R_{0} \geq 0$ are given.

Note that $\mathrm{k}=0,1,2, \ldots, \mathrm{T}-1$, is the index for the time steps. The transitions between different states are described by the following parameters:

$$
\begin{aligned}
& \Lambda \quad \text { is the recruitment rate of susceptible; } \\
& \beta \quad \text { is the effective contact rate; } \\
& \mu \quad \text { is the natural mortality rate; } \\
& \mathrm{d} \quad \text { is the disease induced death rate; } \\
& \mathrm{r} \quad \text { is the recovery rate; } \\
& \alpha_{1}, \alpha_{2} \text { and } \alpha_{3} \text { are positive constants. }
\end{aligned}
$$

Since R does not appear in the first two equations, it is sufficient to analyze the behavior of solutions considering the first two equation of the system (2).

$$
\left\{\begin{array}{l}
S_{k+1}=\Lambda-(\mu-1) S_{k}-\frac{\beta S_{k} I_{k}}{1+\alpha_{1} S_{k}+\alpha_{2} I_{k}+\alpha_{3} S_{k} I_{k}} \\
I_{k+1}=\frac{\beta S_{k} I_{k}}{1+\alpha_{1} S_{k}+\alpha_{2} I_{k}+\alpha_{3} S_{k} I_{k}}-(\mu+d+r-1) I_{k}
\end{array}\right.
$$

The basic reproduction number of system (2), denoted by $\bar{R}_{0}$ is given by

$$
\bar{R}_{0}=\frac{\beta \Lambda}{\left(\mu+\alpha_{1} \Lambda\right)(\mu+d+r)}
$$

We study the local behavior of the model around each of the fixed points. So, we compute the variation matrix corresponding to each fixed point in order to study the local stability analysis of the model above.

The jacobian matrix of this system at an arbitrary point $\mathrm{E}(\mathrm{S}, \mathrm{I})$ is described as follows

$$
J(E)=\left[\begin{array}{cc}
-(\mu-1)-\frac{\beta I_{k}\left(1+\alpha_{2} I_{k}\right)}{T_{k}^{2}} & -\frac{\beta S_{k}\left(1+\alpha_{1} S_{k}\right)}{T_{k}^{2}} \\
\frac{\beta I_{k}\left(1+\alpha_{2} I_{k}\right)}{T_{k}^{2}} & \frac{\beta S_{k}\left(1+\alpha_{1} S_{k}\right)}{T_{k}^{2}}-(\mu+d+r-1)
\end{array}\right]
$$

Where $\mathrm{T}_{\mathrm{k}}=1+\alpha_{1} \mathrm{~S}_{\mathrm{k}}+\alpha_{2} \mathrm{I}_{\mathrm{k}}+\alpha_{3} \mathrm{~S}_{\mathrm{k}} \mathrm{I}_{\mathrm{k}}$. 


\section{STABILITY OF DISEASE-FREE EQUILIBRIUM}

The system (2) always has disease-free fixed point $\mathrm{E}_{\mathrm{f}}\left(\frac{\Lambda}{\mu}, 0\right)$. Then, the Jacobian matrix of this model at this point is given by

$$
J\left(E_{f}\right)=\left[\begin{array}{cc}
-(\mu-1) & -\bar{R}_{0}(\mu+d+r) \\
0 & \left(\bar{R}_{0}-1\right)(\mu+d+r)+1
\end{array}\right]
$$

The two eigenvalues of $\mathbf{J}\left(\mathrm{E}_{\mathrm{f}}\right)$ are $\lambda_{1}=1-\mu$ and $\lambda_{2}=\overline{\mathrm{R}}_{0}-1(\mu+\mathrm{d}+\mathrm{r})+1$.

\section{Theorem 3.1.}

a) $E_{f}$ is locally asymptotically stable if and only if

$$
1-\frac{2}{\mu+d+r}<\bar{R}_{0}<1
$$

b) $E_{f}$ is not locally unstable.

c) $E_{f}$ is saddle if and only if

$$
\overline{\mathrm{R}}_{0}<1-\frac{2}{\mu+\mathrm{d}+\mathrm{r}} \text { or } \overline{\mathrm{R}}_{0}>1
$$

d) $E_{f}$ is non hyperbolic if and only if $\bar{R}_{0}=1$ or $\bar{R}_{0}=1-\frac{2}{\mu+d+r}$.

\section{Proof.}

Let $\lambda_{1}$ and $\lambda_{2}$ be two eigenvalues of matrix $\mathrm{J}(\mathrm{E})$.

We recall some definitions of topological types for a fixed point $E(S, I)$ :

(i) $\quad \mathrm{E}(\mathrm{S}, \mathrm{I})$ is called a sink if $\left|\lambda_{1}\right|<1$ and $\left|\lambda_{2}\right|<1$, so the sink is locally asymptotically stable.

(ii) $\quad \mathrm{E}(\mathrm{S}, \mathrm{I})$ is called a source if $\left|\lambda_{1}\right|>1$ and $\left|\lambda_{2}\right|>1$, so the source is locally unstable.

(iii) $\quad \mathrm{E}(\mathrm{S}, \mathrm{I})$ is called a saddle if $\left|\lambda_{1}\right|<1$ and $\left|\lambda_{2}\right|>1$ or $\left(\left|\lambda_{1}\right|>1\right.$ and $\left.\left|\lambda_{2}\right|<1\right)$.

(iv) $\quad \mathrm{E}(\mathrm{S}, \mathrm{I})$ is non-hyperbolic if either $\left|\lambda_{1}\right|=1$ or $\left|\lambda_{2}\right|=1$

Then

a) $\mu$ is the natural mortality rate, so can't reach 1 . Then $\left|\lambda_{1}\right|<1$ is valid all time.

$\left|\lambda_{2}\right|<1$ if and only if $\left|\left(\bar{R}_{0}-1\right)(\mu+d+r)+1\right|<1$ if and only if $1-\frac{2}{\mu+d+r}<\bar{R}_{0}<1$.

b) $\left|\lambda_{1}\right|>1$ isn't possible, so $E_{f}$ is not locally unstable.

c) $\left|\lambda_{1}\right|<1$ is valid all time. $\left|\lambda_{2}\right|>1$ if and only if $\overline{\mathrm{R}}_{0}<1-\frac{2}{\mu+\mathrm{d}+\mathrm{r}}$ or $\overline{\mathrm{R}}_{0}>1$.

d) $\left|\left(\bar{R}_{0}-1\right)(\mu+d+r)+1\right|<1$ if and only if $\bar{R}_{0}=1$ or $\bar{R}_{0}=1-\frac{2}{\mu+d+r}$.

\section{STABILITY OF ENDEMIC EQUILIBRIUM}

The system has endemic steady state $\mathbf{E}^{*}\left(\mathbf{S}^{*}, \mathbf{I}^{*}\right)$ which exists when $\bar{R}_{0}>1$ and satisfies the following constraints (8) and (9).

$$
\begin{gathered}
I^{*}=a+b S^{*} \\
\text { where } a=\frac{\Lambda}{\mu+d+r} \text { and } b=\frac{-\mu}{\mu+d+r} . \\
A S^{* 2}+B S^{*}+C=0
\end{gathered}
$$

Where $A=-\alpha_{3} \mu$

$$
\begin{aligned}
& B=-\alpha_{1}(\mu+d+r)+\alpha_{3} \Lambda-\alpha_{2} \mu-\beta \\
& C=\mu+d+r+\alpha_{2} \Lambda
\end{aligned}
$$

The discriminant $\Delta=\mathrm{B}^{2}-4 \mathrm{AC}$ 


\section{Theorem 4.1}

If $\alpha_{3}=0$, the system (2) has endemic steady state $E^{*}(-C / B, a-b C / B)$.

If $\alpha_{3} \neq 0$, the system (2) has endemic steady state as long as $|\mathrm{B}|<\sqrt{ } \Delta$.

Furthermore, this endemic steady state $\mathrm{S}_{1} *$ is single and $\mathrm{S}_{1} *=(-\mathrm{B}-\sqrt{ } \Delta) / 2 \mathrm{~A}$.

\section{Proof.}

The epidemiology nature of the system imposes the positivity of susceptible then $S^{*}>0$.

If $\mathrm{S}^{*}=0$ then $\mathrm{C}=0$ (contradiction), so $\mathrm{S}^{*}>0$.

If $\alpha_{3}=0$ then $\mathrm{A}=0$ and $\mathrm{S} *=-\mathrm{C} / \mathrm{B}$.

If $\alpha_{3} \neq 0$, we have two solutions $S_{1} *=(-B-\sqrt{ } \Delta) / 2 A$ and $S_{2} *=(-B+\sqrt{ } \Delta) / 2 A$.

We have $S_{2} *>0$ and $A<0$ so $(-B+\sqrt{ } \Delta)<0$ then $-4 A C<0$ (contradiction), we deduce $B<\sqrt{ } \Delta$ and $\mathrm{S}_{2} *$ is not solution. We have $\mathrm{S}_{1} *>0$ and $\mathrm{A}<0$ so $\mathrm{B}>-\sqrt{ } \Delta$.

\section{Theorem 4.2}

Assume that $\bar{R}_{0}>1$. $E^{*}$ is asymptotically stable if the following condition is satisfied

$$
0 \prec C^{*}+\mu^{2} \prec \mu(2+d+r)
$$

Where

$$
C^{*}=\beta^{2} \frac{\left[\left(S^{*}+\alpha_{1} S^{* 2}\right)^{2}-\left(I^{*}+\alpha_{2} I^{* 2}\right)^{2}\right]}{\left(1+\alpha_{1} S^{*}+\alpha_{2} I^{*}+\alpha_{3} S^{*} I^{*}\right)^{4}}
$$

\section{Proof.}

The characteristic equation of Jacobian matrix $J\left(E^{*}\right)$ is

$$
P(\lambda)=\lambda^{2}+p \lambda+q=0
$$

Where

$$
\begin{aligned}
p & =2 \mu+d+r-2 \\
q & =C^{*}+\mu(p-\mu)+1 \\
C^{*} & =\beta^{2} \frac{\left[\left(s^{*}+\alpha_{1} S^{* 2}\right)^{2}-\left(I^{*}+\alpha_{2} I^{* 2}\right)^{2}\right]}{\left(1+\alpha_{1} S^{*}+\alpha_{2} I^{*}+\alpha_{3} S^{*} I^{*}\right)^{4}}
\end{aligned}
$$

From the Schur-Cohn criterion [13], the zeros of the characteristic polynomial (12) lie inside the unit disk if and only if

$$
\begin{aligned}
& P(1)=1+p+q \succ 0 . \\
& P(-1)=1-p+q \succ 0 . \\
& 1-q \succ 0 .
\end{aligned}
$$

In this case

If $1+p+q>0$, then

$$
C^{*} \succ-\left[\mu^{2}+(d+r)(\mu+1)\right]
$$

If $1-p+q>0$, then

$$
C^{*} \succ-\left[\mu^{2}+(1-\mu)(4-d-r)\right]
$$

If $l-q>0$, then

$$
C^{*} \prec \mu(2-\mu+d+r)
$$

The three conditions above are satisfied if we have (10). 


\section{CONClusion}

In this work, we have established criteria on stability of disease free equilibria depending on reproduction number $\bar{R}_{0}$, otherwise, $E_{f}$ is locally asymptotically stable if and only if $1-\frac{2}{\mu+d+r}<\bar{R}_{0}<1$. On the other side, we prove that $\mathrm{E}^{*}\left(\mathrm{~S}^{*}=-\mathrm{C} / \mathrm{B}, \mathrm{I}^{*}=\mathrm{a}-\mathrm{bC} / \mathrm{B}\right)$ if $\alpha_{3}=0$.

The system has single endemic steady state $S_{1} *=(-B-\sqrt{ } \Delta) / 2 \mathrm{~A}$ if $\quad \alpha_{3} \neq 0$ and $\mathrm{B}<\sqrt{ } \Delta$. We have also determined criterion of stability (10) concerning endemic equilibrium. Finally, the work in this paper contributes to a growing literature on applying stability techniques to epidemiology.

\section{REFERENCES}

[1] L. Zhou, M. Fan, Dynamics of an SIR epidemic model with limited medical resources revisited, Nonlinear Anal. RWA13 312-324, 2012.

[2] Z. W. Jia, G. Y. Tang, Z. Jin et al., Modeling the impact of immigration on the epidemiology of tuberculos, Theoretical Population Biology, vol. 73, no. 3, pp.437-448, 2008.

[3] H. Ibrahim, I. Ahmed, J. Peter Witbooi, and Kailash Patidar, Modeling the Dynamics of an Epidemic under Vaccination in Two Interacting Populations, Journal of Applied Mathematics, article ID 275902, p. 14, 2012.

[4] Y. Enatsu, Y. Nakata, Y. Muroya, G. Izzo, A. Vecchio, Global dynamics of difference equations for SIR epidemic models with a class of nonlinear incidence rates, J. Differ. Equ. Appl, 2011. doi:10.1080/10236198.2011.555405.

[5] S. Jang, On a discrete West Nile epidemic model, Comput. Appl. Math. 26(3), 397-414, 2007.

[6] G. Cruz-Pacheco, L. Esteva, J. Montano-Hirose, C. Vargas, Modelling the dynamics of West Nile virus, Bull. Math. Biol. 67, 1157-1172, 2005.

[7] S. Jang, S. Elaydi, Difference equations from discretization of a continuous epidemic model with immigration of infectives, Canadian Applied Math Quarterly, 11, 93-105, 2003.

[8] A. Kaddar, On the dynamics of a delayed sir epidemic model with a modified saturated incidence rate, Electronic Journal of Differential Equations, vol. 2009, no.133, pp.1-7, 2009.

[9] P. Das, D. Mukherjee and Y. H. Hsieh, An S-I epidemic model with saturation incidence: discrete and stochastic version, International Journal of Nonlinear Analysis and Applications, pp.1-9, 2011.

[10] P. H. Crowley and E. K. Martin, Functional responses and interference within and between year classes of a dragony population, Journal of the North American Benthological Society, vol. 8, pp. 211-221, 1989.

[11] X. Zhou and J. Cui, Global stability of the viral dynamics with crowley-martin functional response, Bulletin of the Korean Mathematical Society, vol. 48, no. 3, pp. 555-574, 2011.

[12] X. Q. Liu, S. M. Zhong, B. D. Tian, and F. X. Zheng, Asymptotic properties of a stochastic predator-prey model with Crowley-Martin functional response, Journal of Applied Mathematics and Computing, 2013.

[13] E. Jury, Theory and Applications of the Z-transform, Wiley, New York, 1964. 